\title{
Elevated CA 19-9 Levels Observed in Association with a Pulmonary Neuroendocrine Tumour and Amyloid
}

\author{
Shoaib Faruqi ${ }^{1}$, Krishna Gumparthy ${ }^{2}$ and Zaroug Wahbi ${ }^{3}$
}

\begin{abstract}
Investigations completed in a 77-year-old ex-smoker presenting with weight loss showed raised CA 19-9 levels. The findings of chest radiograph, abdominal computed tomography (CT) and gastrointestinal endoscopic examinations were all normal. On follow-up, the patient developed left upper lobe collapse on chest radiograph with increasing CA 19-9 levels. Chest CT findings suggested the presence of a left upper lobe tumour. The results of a biopsy of the left upper lobe nodule seen on bronchoscopy suggested a diagnosis of amyloidosis; however, this was not the only diagnosis. The left upper lobe mass comprised a neuroendocrine tumour with amyloid deposition just beneath the bronchial epithelium and focally between the nests of the tumour cells. We report and discuss this uncommon association and presentation.
\end{abstract}

Key words: amyloid, tumour markers, CA 19-9

(Intern Med 52: 105-109, 2013)

(DOI: 10.2169/internalmedicine.52.8254)

\section{Case Report}

A 77 -year-old woman who was an ex-smoker of 40 packyears presented with complaints of weight loss and a decreased appetite. She was known to have mild diverticular disease and experienced a disease flare up with an increased stool frequency of 2-3 bowel movements/day on admission. There was no history of haematochezia, malena, cough, expectoration or haemoptysis. She was known to have bronchial asthma and was receiving treatment with inhaled corticosteroids. On examination, she was not found to have any pallor, clubbing or lymphadenopathy. She had a body mass index of 16. Her heart rate was 80 beats/min and her blood pressure was 130/80 $\mathrm{mmHg}$. The findings of a systemic examination were normal.

The initial diagnoses considered included malignancy (possibly gastrointestinal), mal-absorption syndrome and thyroid dysfunction, and investigations were accordingly organised. Other than a serum albumin level of $2.7 \mathrm{~g} / \mathrm{dL}$, the patient's biochemical profile was normal. Liver function tests were within the normal range and showed a bilirubin level of $16 \mathrm{umol} / \mathrm{L}$, an alkaline phosphatase level of $92 \mathrm{IU} / \mathrm{L}$ and an alanine aminotransferase level of 29 IU/L. A full blood count and thyroid function tests were normal. Chest radiograph findings were normal. Sigmoidoscopy and colonoscopy results were normal other than showing mild diverticular disease. Oesophagogastroduodenoscopy findings were normal, as was the subsequent report of a duodenal biopsy. Tumour marker tests showed that the levels of Carbohydrate Antigen 19-9 (CA 19-9) were raised at $300 \mathrm{ku} / \mathrm{L}$ (normal $<40 \mathrm{ku} / \mathrm{L}$ ). The tests were subsequently repeated, and the CA 19-9 level was found to be $326 \mathrm{ku} / \mathrm{L}$. The Carcinoembryonic Antigen, Alpha-fetoprotein and Cancer Antigen 125 levels were normal. Ultrasound and subsequent computed tomography (CT) of the abdomen and pelvis were normal. The diarrhoea resolved, and the patient was feeling better. A dietary opinion was sought, and the patient was started on nutritional supplements. She was discharged three weeks from admission, as nothing specific was found, with plans to follow up with repeat CA 19-9 level measurements.

Two months after discharge the patient was again admitted, as her symptoms of anorexia and weight loss persisted. The diarrhoea had not recurred. An examination of the respiratory system showed decreased air entry in the left upper chest on auscultation. The remainder of the systemic and

${ }^{1}$ Department of Respiratory Medicine, Castle Hill Hospital, UK, ${ }^{2}$ Department of Pathology, Arrowe Park Hospital, UK and ${ }^{3}$ Department of Respiratory Medicine, Arrowe Park Hospital, UK

Received for publication May 29, 2012; Accepted for publication October 3, 2012

Correspondence to Dr. Shoaib Faruqi, sfaruqi@doctors.net.uk and shoaib.faruqi@rediffmail.com 


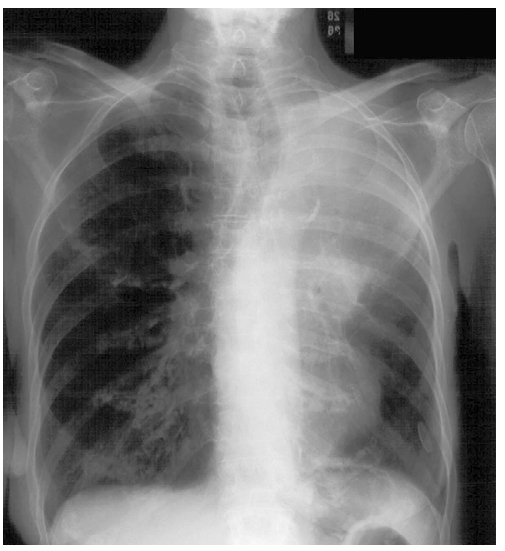

Figure 1. Chest radiograph demonstrating changes in the left upper zone suggestive of left upper lobe collapse.

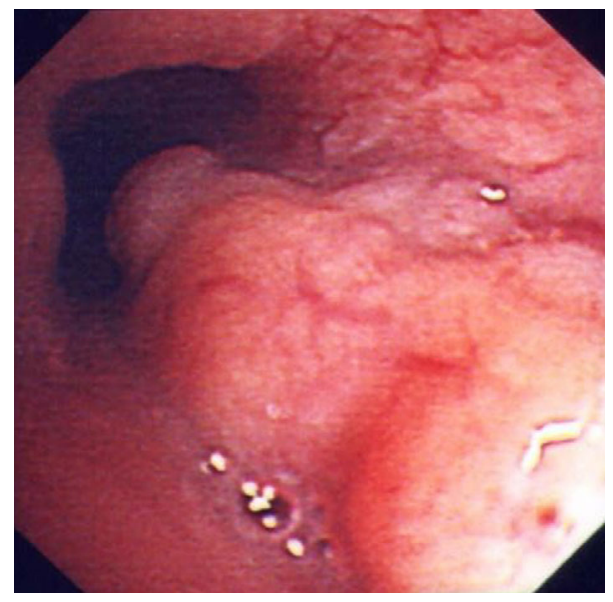

Figure 3. The bronchoscopic appearance of the left main bronchus showing a lumpy lesion at the origin of the left main bronchus.

general examination was normal. The CA 19-9 levels had increased to $1,558 \mathrm{ku} / \mathrm{L}$. The liver function tests remained normal. Chest radiograph was performed, which showed hazy shadowing in the left upper zone suggestive of collapse of the left upper lobe (Fig. 1). With a possible left upper lobe collapse in an ex-smoker with constitutional symptoms, a diagnosis of bronchogenic tumour was considered highly likely. Chest CT showed extensive consolidation in the left upper lobe. There was suspicion of a filling defect in the left main bronchus, as outlined by the calcified bronchial cartilages. The entire proximal left upper lobe bronchus was narrowed and markedly irregular, leading to an abnormal abrupt cut-off of the distal air column. Although no discrete central masses were seen, the appearance was suggestive of a malignant process (Fig. 2). The appearance of both the abdomen and pelvis was normal.

Fibre optic bronchoscopy was performed, which showed areas of pale waxy appearance at the top part of the main carina and the proximal end of the left main bronchus. There was also a pale yellow, large lumpy mass extending down the left main bronchus (Fig. 3). A histopathological examination of the bronchial biopsy showed fragmented res-

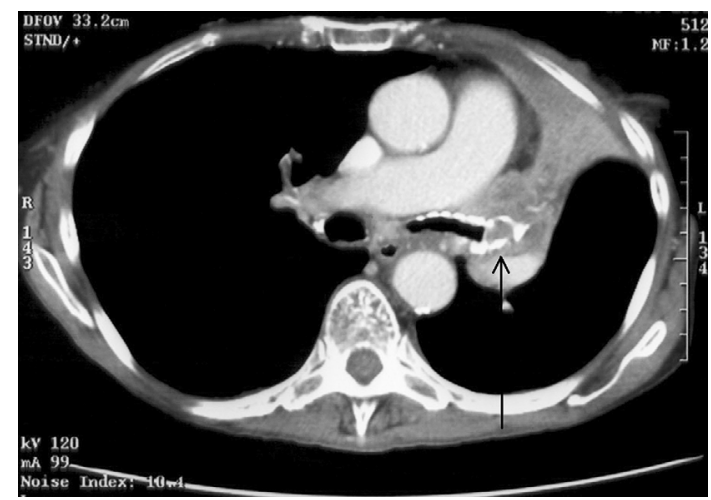

Figure 2. CT image showing left upper lobe consolidation. There was a bronchial filling defect (arrow) outlined by agerelated calcification of the bronchial cartilage. The proximal left upper lobe bronchus appeared narrowed and irregular. No discrete masses were seen.

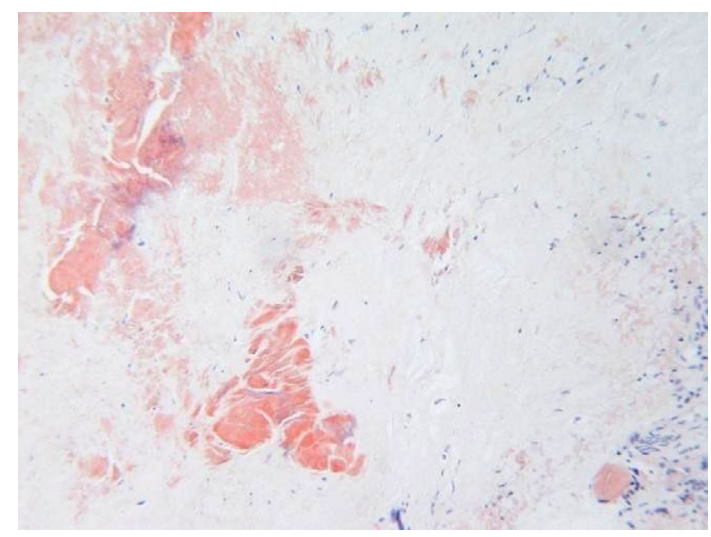

Figure 4. Photomicrograph obtained under low magnification demonstrating orange coloured deposits of amyloid stained with Congo Red. Characteristic apple green birefringence was seen when viewed under cross-polarised light.

piratory mucosa with underlying stroma incorporating homogenous, amorphous, eosinophilic material that was congophilic (Fig. 4). The biopsy showed apple green birefringence characteristic of amyloid. The amyloid subtype was AL Lambda. This suggested a diagnosis of primary tracheobronchial amyloidosis. As this condition is rather rare and the clinical presentation was not characteristic, and in fact suggestive of malignancy, repeat bronchoscopy and biopsies were completed with the same results. A thorough investigation for systemic amyloidosis and plasma cell dyscrasia was conducted. There were no monoclonal bands in the serum or urine. A skeletal survey, bone marrow examination, echocardiogram and rectal biopsy were all normal. Serum amyloid p-component scintigraphy confirmed localised uptake in the region of the left main bronchus. The patient was referred for bronchoscopic laser resection to treat primary tracheobronchial amyloidosis.

Before bronchoscopic laser resection could be undertaken, the patient was again admitted with complete collapse and consolidation of the entire left lung. Earlier, she had experi- 


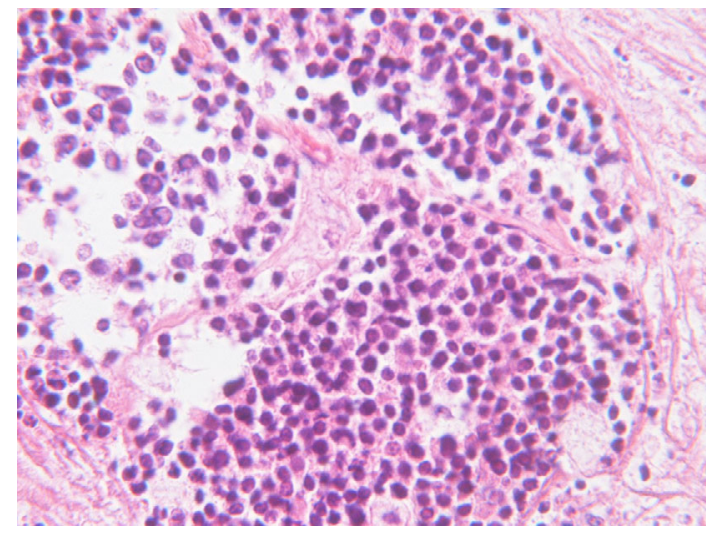

Figure 5. Hematoxylin and Eosin staining photomicrograph obtained under high magnification demonstrating areas of small blue staining neoplastic cells. Immunocytochemistry confirmed these to form a well-differentiated neuroendocrine carcinoma.

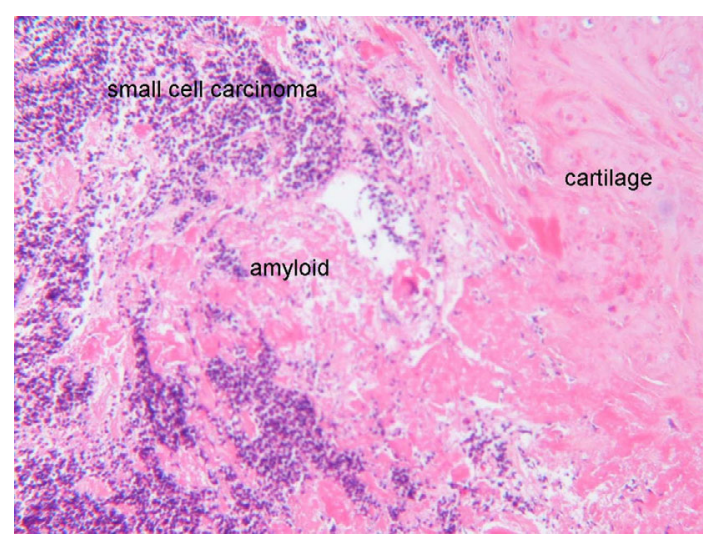

Figure 7. Hematoxylin and Eosin staining photomicrograph obtained under low magnification clearly demonstrating areas of small blue staining neoplastic cells interspersed with uniform eosinophilic amyloid with bronchial cartilage (labelled).

enced two previous chest infections that were managed on an outpatient basis with oral antibiotics. The CA 19-9 level had increased to $3,000 \mathrm{ku} / \mathrm{L}$. The patient was septicaemic, and appropriate treatment was administered. She succumbed to the illness within 72 hours of admission. A complete post-mortem examination was conducted. The histopathological findings are shown in Figs. 5-7.

\section{Discussion}

The first abnormal finding was an elevated CA 19-9 level. CA19-9 has been used as a tumour marker in the diagnosis of pancreatic cancers. It can be used to confirm the diagnosis in suspected symptomatic patients and for prognostication and prediction of recurrence after surgery (1). However, it is neither a very specific nor sensitive marker, and increased levels of CA19-9 have been described in other malignancies, including lung cancers and malignant pleural effusions (2-6). Raised levels of CA 19-9 have been described

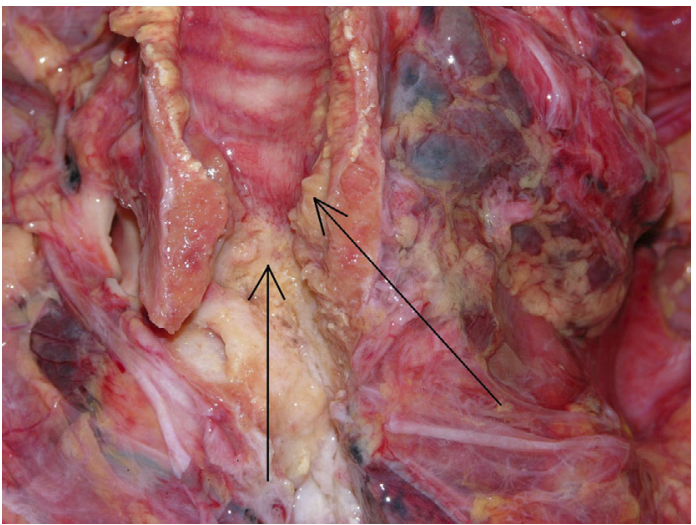

Figure 6. Macroscopic image of a cut section demonstrating a bronchus with the peribronchial tumour and amyloid (pale yellow and waxy, arrows). The amyloid was seen below the bronchial epithelium and between the nests of the tumour cells. Between the bronchial epithelium and the tumour, the amyloid deposit measured up to $20 \mathrm{~mm}$ in thickness.

in a host of benign conditions involving various organ systems such as the hepatobiliary, respiratory, genitourinary and endocrine systems and in rheumatic diseases (7-17). The benign causes of raised CA 19-9 levels are summarised in Table. There are reports of raised CA 19-9 levels in patients with primary amyloidosis as well, although in a different clinical context (18). There are no reports of very high CA 19-9 levels in patients with primary tracheobronchial amyloidosis.

Raised levels of CA 19-9 are observed in several benign respiratory conditions (Table). Our patient did not have any clinical or radiological evidence of pulmonary fibrosis, emphysema, bronchiectasis or lung abscesses. The patient was known to have diverticular disease, and diverticulitis can lead to increased CA 19-9 levels. On presentation, the patient did have diarrhoea; however, it soon resolved. Although benign causes can lead to raised levels of CA 19-9, the increase itself is relatively modest $(10,11)$. Markedly raised levels have usually been described in conjunction with malignant processes. As the patient's CA 19-9 levels were markedly raised, investigations to detect malignancy were undertaken, the results of which were negative along with normal levels of other tumour markers. Hence, it was decided to keep the patient under review and repeat level measurements on follow-up.

Amyloidosis results from extracellular deposition of fibrillar amyloid proteins and is characterised by properties of binding of congo red dye and green birefringence under polarised dye. Amyloidosis can be systemic or localised. Light chain amyloidosis (AL) is the most common type of systemic primary amyloidosis seen in the West. Primary amyloidosis is characterised by the absence of significant plasma cell proliferation, as was observed in this case. Radiolabelled serum amyloid $\mathrm{P}$ component scintigraphy (SAP Scan) is a noninvasive and quantitative method for imaging amyloid deposits. It is a specific technique, complements bi- 
Table. Benign Causes of Raised CA 19-9 Levels

\begin{tabular}{ll}
\hline Hepato-biliary & Pancreatic / Gastro-intestinal \\
\hline Cholecystitis & Acute pancreatitis \\
Cholangitis & Chronic pancreatitis \\
Choledocholithiasis & Pancreatic pseudo-cyst \\
Hepatic cyst / Poly-cystic liver & Diverticulitis \\
Hepatic abscess & \\
Hydatid cyst of the liver & \\
\hline Respiratory & Genito-urinary \\
\hline Pulmonary fibrosis & Seminal vesicle cyst \\
Bronchiectasis & Ovarian cyst \\
Emphysema & Renal cyst \\
Cystic fibrosis & Renal failure \\
Pneumonia & Transverse vaginal septum \\
Benign pleural effusions & \\
\hline Rheumatologic conditions & Other causes \\
\hline Rheumatoid arthritis & Heart failure \\
Systemic lupus erythematosus & Hashimoto's thyroiditis \\
Systemic sclerosis & De Quervain's thyroiditis \\
\hline
\end{tabular}

opsy histology and has been incorporated into suggested algorithms for the evaluation of amyloidosis (19). In this case, a SAP scan was performed, which confirmed localised uptake of amyloid in the region of the left main bronchus.

A diagnosis of localised primary tracheobronchial amyloidosis is uncommon and is limited to small series or case reports (20-25). Amyloid deposits in the walls of the tracheobronchial tree produce submucosal plaques and endobronchial nodules. On bronchoscopic examination, these submucosal plaques appear as smooth, shiny ridges, often causing bronchial narrowing. The endobronchial lesions are polypoid, usually solitary and occur only in major bronchi. Presentation typically occurs after the fifth decade of life and includes dyspnoea, a nonproductive cough and occasionally haemoptysis. Narrowing of the airways can lead to distal atelectasis and recurrent infections and can be mistaken for neoplasias. The prognosis is usually benign; however, recurrent infections can lead to an aggressive clinical course. The treatment options available include debulking, stenting, bronchoscopic ablation, laser resection and external beam radiation (20). In our case, all investigations suggested a diagnosis of tracheobronchial amyloidosis, and appropriate treatment was planned.

A post-mortem examination showed the left lung to be heavy and infiltrated by a yellow, firm and partly necrotic tumour in the upper lobe measuring approximately $8 \mathrm{~cm}$ in maximum diameter. The mass had infiltrated the left main bronchus. The left main bronchus portion of the tumour was both intramural and peribronchial, causing narrowing of the lumen, and extended up to the branching of the left main bronchus into the smaller bronchi. The tumour was also seen to infiltrate the pulmonary arterial wall. The adjoining lung parenchyma was partly collapsed and partly consolidated. The right upper lobe also was consolidated. The histology of the tumour mass was suggestive of a well differentiated neuroendocrine carcinoma. In addition, there were large expansile masses of homogenous eosinophilic material up to $20 \mathrm{~mm}$ in maximal thickness just beneath the bronchial epithelium and focally between the nests of the tumour cells (Figs. 6, 7). The material was congophilic with appropriate birefringence on polarisation consistent with amyloid deposition.

The post-mortem examination showed an interesting picture of a well-differentiated neuroendocrine tumour with amyloid. As the clinical picture was more in keeping with a malignant process, bronchoscopy and biopsies were performed twice. It is likely that the rim of amyloid over the tumour precluded any malignant tissue from being biopsied. This highlights a limitation of bronchoscopic biopsies. A case of a lung nodule comprising adenocarcinoma and amyloid deposition in the context of primary systemic amyloidosis has been previously reported in this journal (26). Pertinent to this case, there is a report of an amyloid-forming "apudoma" of the breast (27). Localized tracheobronchial amyloidosis, if causing atelectasis and recurrent infections, can pursue an aggressive clinical course. Elevated levels of CA 19-9 have been described in patients with tumours of the lung. However, there are no reports of well-differentiated neuroendocrine tumours of the lung leading to markedly raised CA 19-9 levels. Similarly, there are no reports of tracheobronchial amyloidosis leading to very high CA19-9 levels. In the present case, despite conducting appropriate and extensive investigations, the correct diagnosis was elusive.

The authors state that they have no Conflict of Interest (COI).

\section{References}

1. Goonetilleke KS, Siriwardena AK. Systematic review of carbohydrate antigen (CA 19-9) as a biochemical marker in the diagnosis of pancreatic cancer. Eur J Surg Oncol 33: 266-270, 2007.

2. Alatas F, Alatas O, Metintas M, et al. Diagnostic value of CEA, CA 15-3, CA 19-9, CYFRA 21-1, NSE and TSA assay in pleural effusions. Lung Cancer 31: 9-16, 2001.

3. Ohshio G, Yamaki K, Imamura T, et al. Distribution of the carbohydrate antigens, DU-PAN-2 and CA19-9, in tumors of the lung. Tumori 81: 67-73, 1995.

4. Kuralay F, Tokgöz Z, Cömlekci A. Diagnostic usefulness of tumour marker levels in pleural effusions of malignant and benign origin. Clin Chim Acta 300: 43-55, 2000.

5. Rottenberg Y, Nisman B, Peretz T. Extreme high levels of CA19-9 associated with adenocarcinoma of the lung. Isr Med Assoc J 11: 116-117, 2009.

6. Nakajima T, Terashima T, Nishida J, Onoda M, Koide O. Treatment of bronchorrhea by corticosteroids in a case of bronchioloalveolar carcinoma producing CA19-9. Intern Med 41: 225-228, 2002.

7. Shimomura C, Eguchi K, Kawakami A, et al. Elevation of a tumour associated antigen CA 19-9 levels in patients with rheumatic diseases. J Rheumatol 16: 1410-1415, 1989.

8. Parra JL, Kaplan S, Barkin JS. Elevated CA 19-9 caused by Hashimoto's thyroiditis: review of the benign causes of increased CA 19-9 level. Dig Dis Sci 50: 694-695, 2005.

9. Kim HR, Lee CH, Kim YW, Han SK, Shim YS, Yim JJ. Increased CA 19-9 level in patients without malignant disease. Clin Chem Lab Med 47: 750-754, 2009.

10. Pavai S, Yap SF. The clinical significance of elevated levels of serum CA 19-9. Med J Malaysia 58: 667-672, 2003.

11. Ballehaninna UK, Chamberlain RS. The clinical utility of serum CA 19-9 in the diagnosis, prognosis and management of pancre- 
atic adenocarcinoma: an evidence based appraisal. J Gastrointest Oncol 3: 105-119, 2012.

12. Kaya C, Cengiz H, Ekin M, Yasar L. Transverse vaginal septum: a benign reason for elevated serum CA 19-9 and CA 125 levels. Arch Gynecol Obstet 2012(Apr 27, Epub ahead of print).

13. Ramia JM, De la Plaza R, Quiñones J, Veguillas $P$, Garcia-Parreño J. Extremely high values of CA 19-9 in liver hydatidosis and frank biliary rupture. Turk J Gastroenterol 22: 548-550, 2011.

14. Doğan ÜB, Gümürdülü Y, Gölge N, Kara B. Relationship of CA 19-9 with choledocholithiasis and cholangitis. Turk J Gastroenterol 22: 171-177, 2011.

15. Kelly PJ, Archbold P, Price JH, Cardwell C, McCluggage WG. Serum CA 19.9 levels are commonly elevated in primary ovarian mucinous tumours but cannot be used to predict the histological subtype. J Clin Pathol 63: 169-173, 2010.

16. Waanders E, van Keimpema L, Brouwer JT, et al. Carbohydrate antigen 19-9 is extremely elevated in polycystic liver disease. Liver Int 29: 1389-1395, 2009.

17. Ventrucci M, Pozzato P, Cipolla A, Uomo G. Persistent elevation of serum CA 19-9 with no evidence of malignant disease. Dig Liver Dis 41: 357-363, 2009.

18. Mohr A, Miehlke S, Klauck S, et al. P. Hepatomegaly and cholestasis as primary clinical manifestations of an AL-kappa amyloidosis. Eur J Gastroenterol Hepatol 11: 921-925, 1999.
19. Lachmann HJ, Hawkins PN. Systemic amyloidosis. Curr Opin Pharmacol 6: 214-220, 2006.

20. Sharma OP. Paraproteinemias and the lungs. Curr Opin Pulm Med 11: 408-411, 2005.

21. Ding L, Li W, Wang K, et al. Primary tracheobronchial amyloidosis in China: analysis of 64 cases and a review of literature. J Huazhong Univ Sci Technolog Med Sci 30: 599-603, 2010.

22. Ozyigit LP, Kiyan E, Okumus G, Yilmazbayhan D. Isolated laryngo-tracheal amyloidosis presenting as a refractory asthma and longstanding hoarseness. J Asthma 46: 314-317, 2009.

23. Hanon S, De Keukeleire T, Dieriks B, et al. Primary tracheobronchial amyloidosis: a series of 3 cases. Acta Clin Belg 62: 56-60, 2007.

24. Gaurav K, Panda M. An uncommon cause of bilateral pulmonary nodules in a long-term smoker. J Gen Intern Med 22: 1617-1620, 2007.

25. Eguchi T, Yoshida K, Kobayashi N, et al. Localized nodular amyloidosis of the lung. Gen Thorac Cardiovasc Surg 59: 715-717, 2011.

26. Miyazaki D, Yazaki M, Ishii W, et al. A rare lung nodule consisting of adenocarcinoma and amyloid deposition in a patient with primary systemic AL amyloidosis. Intern Med 50: 243-246, 2011.

27. Serezhin BS, Semova NV, Paîkova LV. Amyloid-forming apudoma of the breast. Arkh Patol 47: 57-60, 1985 (in Russian).

(C) 2013 The Japanese Society of Internal Medicine http://www.naika.or.jp/imonline/index.html 